\title{
A new refinement of discrete Jensen's inequality depending on parameters
}

\section{László Horváth*}

\section{"Correspondence:}

Ihorvath@almos.uni-pannon.hu Department of Mathematics, University of Pannonia, Egyetem u. 10, Veszprém, 8200, Hungary

\begin{abstract}
In this paper we give a new refinement of discrete Jensen's inequality, which generalizes a former result. The introduced sequences depend on parameters. The strict monotonicity and the convergence are investigated. We also study the behavior of the sequences when the parameters vary. One of the proofs requires an interesting convergence theorem with probability theoretical background. This result is an extension of a former result, but its proof is simpler. The results are applied to define and study some new quasi-arithmetic means.
\end{abstract}

MSC: 26D07; 26A51

Keywords: convex function; mid-convex function; discrete Jensen's inequality; quasi-arithmetic mean

\section{Introduction}

We begin with discrete Jensen's inequality which is a useful tool in different parts of mathematics. The set of nonnegative integers and that of positive integers will be denoted by $\mathbb{N}$ and $\mathbb{N}_{+}$, respectively.

Theorem A (see [1]) Let $C$ be a convex subset of a real vector space $X$, and let $\left\{x_{1}, \ldots, x_{n}\right\}$ be a finite subset of $C$, where $n \in \mathbb{N}_{+}$is fixed. Let $p_{1}, \ldots, p_{n}$ be nonnegative numbers with $\sum_{j=1}^{n} p_{j}=1$. If $: C \rightarrow \mathbb{R}$ is either a convex or a mid-convex function and in the latter case the numbers $p_{j}(1 \leq j \leq n)$ are rational, then

$$
f\left(\sum_{j=1}^{n} p_{j} x_{j}\right) \leq \sum_{j=1}^{n} p_{j} f\left(x_{j}\right) .
$$

The function $f: C \rightarrow \mathbb{R}$ is called convex if

$$
f(\alpha x+(1-\alpha) y) \leq \alpha f(x)+(1-\alpha) f(y), \quad x, y \in C, 0 \leq \alpha \leq 1,
$$

and mid-convex or Jensen-convex if

$$
f\left(\frac{x+y}{2}\right) \leq \frac{1}{2} f(x)+\frac{1}{2} f(y), \quad x, y \in C
$$

$f$ is strictly convex if strict inequality holds in (2) whenever $x \neq y$ and $0<\alpha<1$.

\section{Springer}

@2013 Horváth; licensee Springer. This is an Open Access article distributed under the terms of the Creative Commons Attribution License (http://creativecommons.org/licenses/by/2.0), which permits unrestricted use, distribution, and reproduction in any medium, provided the original work is properly cited. 
In recent years, a number of papers have appeared in which the authors constructed different refinements of discrete Jensen's inequality. See, for example, [2-8] and [9]. These papers also contain a lot of interesting applications. The following parameter-dependent refinement can be found in [4].

Theorem B Let $C$ be a convex subset of a real vector space $X$, and let $\left\{x_{1}, \ldots, x_{n}\right\}$ be a finite subset of $C$, where $n \in \mathbb{N}_{+}$is fixed. Let $p_{1}, \ldots, p_{n}$ be nonnegative numbers with $\sum_{j=1}^{n} p_{j}=1$, and let $\lambda \geq 1$. Suppose that $f: C \rightarrow \mathbb{R}$ is either a convex or a mid-convex function and in the latter case the numbers $p_{j}(1 \leq j \leq n)$ and $\lambda$ are rational. Introduce the sets

$$
S_{k}:=\left\{\left(i_{1}, \ldots, i_{n}\right) \in \mathbb{N}^{n} \mid \sum_{j=1}^{n} i_{j}=k\right\}, \quad k \in \mathbb{N},
$$

and for $k \in \mathbb{N}$ define the numbers

$$
\begin{aligned}
C_{k}(\lambda) & =C_{k}\left(x_{1}, \ldots, x_{n} ; p_{1}, \ldots, p_{n} ; \lambda\right) \\
& :=\frac{1}{(n+\lambda-1)^{k}} \sum_{\left(i_{1}, \ldots, i_{n}\right) \in S_{k}} \frac{k !}{i_{1} ! \cdots i_{n} !}\left(\sum_{j=1}^{n} \lambda^{i_{j}} p_{j}\right) f\left(\frac{\sum_{j=1}^{n} \lambda^{i_{j}} p_{j} x_{j}}{\sum_{j=1}^{n} \lambda^{i_{j}} p_{j}}\right) .
\end{aligned}
$$

Then:

(a)

$$
f\left(\sum_{j=1}^{n} p_{j} x_{j}\right)=C_{0}(\lambda) \leq C_{1}(\lambda) \leq \cdots \leq C_{k}(\lambda) \leq \cdots \leq \sum_{j=1}^{n} p_{j} f\left(x_{j}\right), \quad k \in \mathbb{N} .
$$

(b) Suppose that $X$ is a normed space and $f$ is continuous. For every fixed $\lambda>1$,

$$
\lim _{k \rightarrow \infty} C_{k}(\lambda)=\sum_{j=1}^{n} p_{j} f\left(x_{j}\right)
$$

Our aim is to present a generalization of the previous result.

The sequence $\left(C_{k}(\lambda)\right)_{k=0}^{\infty}$ depends on one parameter, but we allow the new sequence $\left(D_{k}(\lambda)\right)_{k=0}^{\infty}$ (see (7)) to depend on $n$ parameters. We give conditions for the strict monotonicity of $\left(D_{k}(\lambda)\right)_{k=0}^{\infty}$ in some cases.

The proof of limit assertion (5) uses a lemma (see Lemma 15 in [4]) with a rather difficult probability theoretical proof. Essentially, a fundamental theorem from statistics, which is based on the multidimensional central limit theorem, has been applied. In this work, we obtain a convergence theorem extending (5) which will be deduced from another interesting lemma analogous to Lemma 15 in [4]. The proof of this lemma is simpler than the proof of Lemma 15 in [4], although it also has probability theoretical background, namely the strong law of large numbers is used.

The behavior of the sequence $\left(D_{k}(\lambda)\right)_{k=0}^{\infty}$ is studied when the parameters vary. Further refinements of discrete Jensen's inequality can be derived from these results.

As an application, some new quasi-arithmetic means are constructed, and the monotonicity and convergence of these means are investigated. 


\section{The main results and some preliminary results}

The main results are given by Theorem 1 and Theorem 5 . In order to render the main results as transparently as possible, we also give some preparatory lemmas and theorems.

The first result generalizes Theorem B.

Theorem 1 Let $C$ be a convex subset of a real vector space $X$, and let $\left\{x_{1}, \ldots, x_{n}\right\}$ be a finite subset of $C$, where $n \in \mathbb{N}_{+}$is fixed. Let $p_{1}, \ldots, p_{n}$ be nonnegative numbers with $\sum_{j=1}^{n} p_{j}=$ 1 , and let $\lambda_{i}>1(1 \leq i \leq n)$. Suppose that $f: C \rightarrow \mathbb{R}$ is either a convex or a mid-convex function, and in the latter case, the numbers $p_{i}$ and $\lambda_{i}(1 \leq i \leq n)$ are rational.

For $k \in \mathbb{N}$, introduce

$$
d(\lambda)=d\left(\lambda_{1}, \ldots, \lambda_{n}\right):=\sum_{j=1}^{n} \frac{1}{\lambda_{j}-1}
$$

and define

$$
\begin{aligned}
D_{k}(\lambda)= & D_{k}\left(x_{1}, \ldots, x_{n} ; p_{1}, \ldots, p_{n} ; \lambda_{1}, \ldots, \lambda_{n}\right) \\
:= & \frac{1}{(d(\lambda)+1)^{k}} \\
& \cdot \sum_{\left(i_{1}, \ldots, i_{n}\right) \in S_{k}} \frac{k !}{i_{1} ! \cdots i_{n} !} \prod_{j=1}^{n} \frac{1}{\left(\lambda_{j}-1\right)^{i_{j}}}\left(\sum_{j=1}^{n} \lambda_{j}^{i_{j}} p_{j}\right) f\left(\frac{\sum_{j=1}^{n} \lambda_{j}^{i_{j}} p_{j} x_{j}}{\sum_{j=1}^{n} \lambda_{j}^{i_{j}} p_{j}}\right),
\end{aligned}
$$

where $S_{k}$ means the set in (3).

Then:

(a)

$$
f\left(\sum_{j=1}^{n} p_{j} x_{j}\right)=D_{0}(\lambda) \leq D_{1}(\lambda) \leq \cdots \leq D_{k}(\lambda) \leq \cdots \leq \sum_{j=1}^{n} p_{j} f\left(x_{j}\right), \quad k \in \mathbb{N} .
$$

(b) Assuming $X$ is a normed space and $f$ is continuous, we have

$$
\lim _{k \rightarrow \infty} D_{k}(\lambda)=\sum_{j=1}^{n} p_{j} f\left(x_{j}\right)
$$

The result contains Theorem B as a special case $\left(\lambda=\lambda_{1}=\cdots=\lambda_{n}\right)$. Since $f$ is continuous in part (b) of the previous result, $f$ is necessarily convex in this case.

The clue of the proof of Theorem 1(b) is the following decisive assertion, which is deduced from the strong law of large numbers. This result extends Lemma 15 in [4] with a less complicated proof.

Theorem 2 Let $C$ be a convex subset of a real normed space $X$, and let $\left\{x_{1}, \ldots, x_{n}\right\}$ be a finite subset of $C$, where $n \in \mathbb{N}_{+}$is fixed. Let $p_{1}, \ldots, p_{n}$ be positive numbers with $\sum_{j=1}^{n} p_{j}=1$, and let $\lambda_{i}>1(1 \leq i \leq n)$. Suppose $f: C \rightarrow \mathbb{R}$ is a convex and continuous function. Define, for $l=1, \ldots, n$,

$$
D_{l k}:=\frac{1}{(d(\lambda)+1)^{k}} \sum_{\left(i_{1}, \ldots, i_{n}\right) \in S_{k}} \frac{k !}{i_{1} ! \cdots i_{n} !} \lambda_{l}^{i_{l}} \prod_{j=1}^{n} \frac{1}{\left(\lambda_{j}-1\right)^{i_{j}}} f\left(\frac{\sum_{j=1}^{n} \lambda_{j}^{i_{j}} p_{j} x_{j}}{\sum_{j=1}^{n} \lambda_{j}^{i_{j}} p_{j}}\right) .
$$


Then we have

$$
\lim _{k \rightarrow \infty} D_{l k}=f\left(x_{l}\right), \quad 1 \leq l \leq n .
$$

Next, we determine some of the cases of strict inequality in (8).

Theorem 3 Let $C$ be a convex subset of a real vector space $X$, and let $x_{1}, \ldots, x_{n}$ be points of $C$ with at least two different elements, where either $n=2$ or $n=3$. Let $p_{1}, \ldots, p_{n}$ be positive numbers with $\sum_{j=1}^{n} p_{j}=1$, and let $\lambda_{i}>1(1 \leq i \leq n)$. If $: C \rightarrow \mathbb{R}$ is strictly convex, then every inequality is strict in (8).

Probably, the result remains true if $n>3$, but the method applied in the proof of Theorem 3 is getting more and more chaotic. Another treatment of the problem may be successful.

In the proof of Theorem 3, we shall use the following well-known result (see [1]).

Theorem C Let $C$ be a convex subset of a real vector space $X$, and let $x_{1}, \ldots, x_{n}$ be points of $C$ with at least two different elements, where $n \geq 2$ is a fixed integer. Let $p_{1}, \ldots, p_{n}$ be positive numbers with $\sum_{j=1}^{n} p_{j}=1$. Iff $: C \rightarrow \mathbb{R}$ is strictly convex, then the inequality is strict in (1).

The following notations and observations may help to understand the next results, and they will be used in their proofs. We motivate proceeding in this direction as follows: consider Theorem 1 , and let $I:=\left\{i \in\{1, \ldots, n\} \mid p_{i} \neq 0\right\}$. It is easy to see that only the restriction of $f$ to the convex hull of $\left\{x_{i} \mid i \in I\right\}$ has significance.

Let $C$ be a convex subset of a real vector space $X$, and let $\left\{x_{1}, \ldots, x_{n}\right\}$ be a finite subset of $C$, where $n \geq 2$ is a fixed integer. Suppose that $f: C \rightarrow \mathbb{R}$ is a convex function. Choose the integers $l \in\{1, \ldots, n\}$ and $1 \leq l_{1}<l_{2}<\cdots<l_{m} \leq n$, where $m \leq n-1$ and $l_{i} \neq l(i=1, \ldots, m)$. Define the convex set

$$
G_{m}:=\left\{\left(t_{1}, \ldots, t_{m}\right) \in \mathbb{R}^{m} \mid t_{i} \geq 0(i=1, \ldots, m), \sum_{i=1}^{m} t_{i} \leq 1\right\}
$$

and the function $h_{l_{1}, \ldots, l_{m}}$ on $G_{m}$ by

$$
h_{l_{1}, \ldots, l_{m}}\left(t_{1}, \ldots, t_{m}\right):=f\left(\sum_{i=1}^{m} t_{i} x_{l_{i}}+\left(1-\sum_{i=1}^{m} t_{i}\right) x_{l}\right) \text {. }
$$

Then $h_{l_{1}, \ldots, l_{m}}$ is well defined since the set

$$
\left\{\sum_{i=1}^{m} t_{i} x_{l_{i}}+\left(1-\sum_{i=1}^{m} t_{i}\right) x_{l} \in X \mid\left(t_{1}, \ldots, t_{m}\right) \in G_{m}\right\}
$$

is the convex hull of $\left\{x_{l_{1}}, \ldots, x_{l_{m}}, x_{l}\right\}$, and hence it is a subset of $C$. A consequence of the convexity of $f$ is that $h_{l_{1}, \ldots, l_{m}}$ is also convex. Further properties of the function $h_{l_{1}, \ldots, l_{m}}$ are studied in the following lemma.

Lemma 4 Let $C$ be a convex subset of a real vector space $X$, and let $\left\{x_{1}, \ldots, x_{n}\right\}$ be a finite subset of $C$, where $n \geq 2$ is a fixed integer. Suppose that $f: C \rightarrow \mathbb{R}$ is a convex function. 
Choose the integers $l \in\{1, \ldots, n\}$ and $1 \leq l_{1}<l_{2}<\cdots<l_{m} \leq n$, where $m \leq n-1$ and $l_{i} \neq l$ $(i=1, \ldots, m)$. Then:

(a) $h_{l_{1}, \ldots, l_{m}}$ is continuous on the interior $\operatorname{int}\left(G_{m}\right)$ of $G_{m}$.

(b) $h_{l_{1}, \ldots, l_{m}}$ is bounded.

(c) The restriction of $h_{l_{1}, \ldots, l_{m}}$ to $\operatorname{int}\left(G_{m}\right)$ has a unique continuous (and also convex) extension $\bar{h}_{l_{1}, \ldots, l_{m}}$ to $G_{m}$.

There are some trivial cases of Theorem 1 : if $p_{l}=1$ for an $l \in\{1, \ldots, n\}$, then

$$
D_{k}(\lambda)=f\left(x_{l}\right), \quad k \in \mathbb{N},
$$

and therefore $0 \leq p_{j}<1(1 \leq j \leq n)$ (thus $\left.n \geq 2\right)$ will be supposed in the next main result, in which the parameter $\lambda=\left(\lambda_{1}, \ldots, \lambda_{n}\right)$ varies.

Theorem 5 Let $C$ be a convex subset of a real vector space $X$, and let $\left\{x_{1}, \ldots, x_{n}\right\}$ be a finite subset of $C$, where $n \geq 2$ is a fixed integer. Let $p_{1}, \ldots, p_{n}$ be nonnegative numbers such that $p_{j}<1(1 \leq j \leq n)$ and $\sum_{j=1}^{n} p_{j}=1$, and let $\lambda_{i}>1(1 \leq i \leq n)$. Suppose that $f: C \rightarrow \mathbb{R}$ is a convex function. Choose $l \in\{1, \ldots, n\}$. Then, for every fixed $\lambda_{i}(1 \leq i \leq n, i \neq l)$ and for each fixed $k \in \mathbb{N}_{+}$:

(a)

$$
\lim _{\lambda_{l} \rightarrow 1+} D_{k}(\lambda)=f\left(\sum_{j=1}^{n} p_{j} x_{j}\right) .
$$

$\left(\mathrm{b}_{1}\right)$ In the case $p_{l}=0$,

$$
\begin{aligned}
\lim _{\lambda_{l} \rightarrow \infty} D_{k}(\lambda)= & \frac{1}{\left(\sum_{\substack{j=1 \\
j \neq l}}^{n} \frac{1}{\lambda_{j}-1}+1\right)^{k}} \\
& \cdot \sum_{\substack{\left(i_{1}, \ldots, i_{n}\right) \in S_{k} \\
i_{l}=0}} \frac{k !}{i_{1} ! \cdots i_{n} !} \prod_{j=1}^{n} \frac{1}{\left(\lambda_{j}-1\right)^{i_{j}}}\left(\sum_{j=1}^{n} \lambda_{j}^{i_{j}} p_{j}\right) f\left(\frac{\sum_{j=1}^{n} \lambda_{j}^{i_{j}} p_{j} x_{j}}{\sum_{j=1}^{n} \lambda_{j}^{i_{j}} p_{j}}\right) .
\end{aligned}
$$

$\left(\mathrm{b}_{2}\right)$ In the case $0<p_{l}(<1)$, there exists an integer $1 \leq m \leq n-1$ such that $m$ elements of the sequence $p_{1}, \ldots, p_{l-1}, p_{l+1}, \ldots, p_{n}$ belong to the open interval $] 0,1[$, and $n-1-m$ members are 0 . We can assume that $\left.p_{l_{1}}, \ldots, p_{l_{m}} \in\right] 0,1\left[\left(1 \leq l_{1}<l_{2}<\cdots<l_{m} \leq n\right.\right.$ and they are different from $l$ ) and the others are 0. By using the function $\bar{h}_{l_{1}, \ldots, l_{m}}$ (see (10) and Lemma 4(c)), we have

$$
\begin{aligned}
\lim _{\lambda_{l} \rightarrow \infty} D_{k}(\lambda)= & \frac{1}{\left(\sum_{\substack{j=1 \\
j \neq l}}^{n} \frac{1}{\lambda_{j}-1}+1\right)^{k}} \\
& \cdot\left(\sum_{\substack{\left(i_{1}, \ldots, i_{n}\right) \in S_{k} \\
i_{l}=0}} \frac{k !}{i_{1} ! \cdots i_{n} !} \prod_{j=1}^{n} \frac{1}{\left(\lambda_{j}-1\right)^{i_{j}}}\left(\sum_{j=1}^{n} \lambda_{j}^{i_{j}} p_{j}\right) f\left(\frac{\sum_{j=1}^{n} \lambda_{j}^{i_{j}} p_{j} x_{j}}{\sum_{j=1}^{n} \lambda_{j}^{i_{j}} p_{j}}\right)\right. \\
& \left.+p_{l} \bar{h}_{l_{1}, \ldots, l_{m}}(0, \ldots, 0)\left(\left(\sum_{\substack{j=1 \\
j \neq l}}^{n} \frac{1}{\lambda_{j}-1}+1\right)^{k}-\left(\sum_{\substack{j=1 \\
j \neq l}}^{n} \frac{1}{\lambda_{j}-1}\right)^{k}\right)\right) .
\end{aligned}
$$


By (8), the limits in (11) and (13) also refine discrete Jensen's inequality. It should be mentioned that in Theorem 1 the topology on $X$ is needed in showing (b), but Theorem 5 is true without any topology on $X$.

\section{Applications}

As an application we can now extend the quasi-arithmetic means introduced in [4] (about means, see [10]).

Definition 6 Let $I \subset \mathbb{R}$ be an interval, $x_{j} \in I(1 \leq j \leq n), p_{1}, \ldots, p_{n}$ be nonnegative numbers such that $\sum_{j=1}^{n} p_{j}=1$, and let $\lambda_{j}>1(1 \leq j \leq n)$. Let $\varphi, \psi: I \rightarrow \mathbb{R}$ be continuous and strictly monotone functions. We define the quasi-arithmetic means with respect to (7) by

$$
\begin{aligned}
M_{\psi, \varphi}^{\lambda}(k):= & \psi^{-1}\left(\frac{1}{(d(\lambda)+1)^{k}} \sum_{\left(i_{1}, . ., i_{n}\right) \in S_{k}} \frac{k !}{i_{1} ! \cdots i_{n} !} \prod_{j=1}^{n} \frac{1}{\left(\lambda_{j}-1\right)^{i_{j}}}\left(\sum_{j=1}^{n} \lambda_{j}^{i_{j}} p_{j}\right)\right. \\
& \left.\cdot\left(\psi \circ \varphi^{-1}\right)\left(\frac{\sum_{j=1}^{n} \lambda_{j}^{i_{j}} p_{j} \varphi\left(x_{j}\right)}{\sum_{j=1}^{n} \lambda_{j}^{i_{j}} p_{j}}\right)\right), \quad k \in \mathbb{N} .
\end{aligned}
$$

We study the monotonicity and convergence of the new means. For this, the next mean is also needed.

Definition 7 Let $I \subset \mathbb{R}$ be an interval, $x_{j} \in I(1 \leq j \leq n), p_{1}, \ldots, p_{n}$ be nonnegative numbers such that $\sum_{j=1}^{n} p_{j}=1$. For a continuous and strictly monotone function $z: I \rightarrow \mathbb{R}$, we introduce the following mean:

$$
M_{z}:=z^{-1}\left(\sum_{j=1}^{n} p_{j} z\left(x_{j}\right)\right) .
$$

We now prove the monotonicity of the means (14) and give limit formulas.

Proposition 8 Let $I \subset \mathbb{R}$ be an interval, $x_{j} \in I(1 \leq j \leq n), p_{1}, \ldots, p_{n}$ be nonnegative numbers such that $\sum_{j=1}^{n} p_{j}=1$, and let $\lambda_{j}>1(1 \leq j \leq n)$. Let $\varphi, \psi: I \rightarrow \mathbb{R}$ be continuous and strictly monotone functions. Then:

(a)

$$
M_{\varphi}=M_{\psi, \varphi}^{\lambda}(0) \leq \cdots \leq M_{\psi, \varphi}^{\lambda}(k) \leq \cdots \leq M_{\psi}, \quad k \in \mathbb{N}
$$

if either $\psi \circ \varphi^{-1}$ is convex and $\psi$ is increasing or $\psi \circ \varphi^{-1}$ is concave and $\psi$ is decreasing.

(b)

$$
M_{\varphi}=M_{\psi, \varphi}^{\lambda}(0) \geq \cdots \geq M_{\psi, \varphi}^{\lambda}(k) \geq \cdots \geq M_{\psi}, \quad k \in \mathbb{N}
$$

if either $\psi \circ \varphi^{-1}$ is convex and $\psi$ is decreasing or $\psi \circ \varphi^{-1}$ is concave and $\psi$ is increasing.

(c) Moreover, in both cases

$$
\lim _{k \rightarrow \infty} M_{\psi, \varphi}^{\lambda}(k)=M_{\psi} .
$$


Proof Theorem 1(a) can be applied to the function $\psi \circ \varphi^{-1}$ if it is convex $\left(-\psi \circ \varphi^{-1}\right.$ if it is concave) and to the $n$-tuples $\left(\varphi\left(x_{1}\right), \ldots, \varphi\left(x_{n}\right)\right)$, then upon taking $\psi^{-1}$, we get (a) and (b). (c) Comes from Theorem 1(b).

As an illustration, we consider the following special case.

Example 9 If $I:=] 0, \infty[, \psi:=\ln$ and $\varphi(x):=x(x \in] 0, \infty[)$, then by Proposition $8(\mathrm{~b})$, we have the following sharpened version of the weighted arithmetic mean-geometric mean inequality: for every $x_{j}>0(1 \leq j \leq n), \lambda_{j}>1(1 \leq j \leq n)$ and $k \in \mathbb{N}_{+}$,

$$
\begin{aligned}
\sum_{j=1}^{n} p_{j} x_{j} & \geq \prod_{\left(i_{1}, \ldots, i_{n}\right) \in S_{k}}\left(\frac{\sum_{j=1}^{n} \lambda^{i_{j}} p_{j} x_{j}}{\sum_{j=1}^{n} \lambda^{i_{j}} p_{j}}\right)^{\frac{1}{(d(\lambda)+1)^{k}} \frac{k !}{i_{1} ! \cdots i_{n} !} \prod_{j=1}^{n} \frac{1}{\left(\lambda_{j}-1\right)^{i_{j}}}\left(\sum_{j=1}^{n} \lambda_{j}^{i_{j}} p_{j}\right)} \\
& \geq \prod_{j=1}^{n} x_{j}^{p_{j}} .
\end{aligned}
$$

\section{Proofs and some auxiliary results}

Before giving the proof of the main result, we introduce some preliminary lemmas. First, a simple inequality is established.

Lemma 10 If $x, y>1$, then

$$
\frac{\ln (y)}{y-1}-\frac{x \ln (x)}{x-1}<0 .
$$

Proof We shall show that

$$
\frac{\ln (y)}{y-1}<1<\frac{x \ln (x)}{x-1}, \quad x, y>1 .
$$

The left inequality is well known, and the right inequality is equivalent to

$$
u(x)=\ln (x)-1+\frac{1}{x}>0, \quad x>1 .
$$

Since

$$
u^{\prime}(x)=\frac{x-1}{x^{2}}>0, \quad x>1,
$$

$u$ is strictly increasing on $[1, \infty)$, hence $u(x)>u(1)=0(x>1)$.

In the second lemma, the measurability of some functions is studied.

Lemma 11 Let $(\Omega, \mathcal{A})$ be a measurable space, and let $X$ be a real normed space. $\mathcal{B}$ denotes the $\sigma$-algebra of Borel sets in $X$. If $A \in \mathcal{A}, v: A \rightarrow \mathbb{R}$ is measurable, and $x \in X$ is fixed, then the function

$$
\omega \rightarrow v(\omega) x, \quad \omega \in A
$$

is $\mathcal{A}-\mathcal{B}$ measurable. 
Proof Since $X$ is a normed space, the mapping $p: \mathbb{R} \rightarrow X, p(c)=c x$ is continuous. Now the result follows from the fact that function (16) can be written as $p \circ v$.

After these preparations, we can now prove Theorem 2.

Proof of Theorem 2 We can obviously suppose that $n \geq 2$ and $l=1$.

Let

$$
q_{1}:=\frac{\lambda_{1}}{(d(\lambda)+1)\left(\lambda_{1}-1\right)}, \quad q_{j}:=\frac{1}{(d(\lambda)+1)\left(\lambda_{j}-1\right)}, \quad 2 \leq j \leq n .
$$

Then $q_{j}>0(1 \leq j \leq n)$ and $\sum_{j=1}^{n} q_{j}=1$, that is, we have a discrete distribution.

Let $(\Omega, \mathcal{A}, P)$ be a probability space, and let

$$
\chi_{k}: \Omega \rightarrow \mathbb{R}, \quad k \in \mathbb{N}_{+}
$$

be identically distributed and independent random variables such that

$$
P\left(\chi_{k}=j\right)=q_{j}, \quad 1 \leq j \leq n, k \in \mathbb{N}_{+} .
$$

Define the random variables

$$
Y_{j k}:=\sum_{t=1}^{k} 1_{\left\{\chi_{t}=j\right\}}, \quad 1 \leq j \leq n, k \in \mathbb{N}_{+},
$$

where $1_{A}: \Omega \rightarrow \mathbb{R}$ means the characteristic function of the set $A \subset \Omega$. The joint distribution of the vector random variable $\left(Y_{1 k}, \ldots, Y_{n k}\right)$ is a multinomial distribution

$$
P\left(Y_{1 k}=i_{1}, \ldots, Y_{n k}=i_{n}\right)=\frac{k !}{i_{1} ! \cdots i_{n} !} q_{1}^{i_{1}} \cdots q_{n}^{i_{n}}, \quad\left(i_{1}, \ldots, i_{n}\right) \in S_{k}
$$

for every $k \in \mathbb{N}_{+}$.

Lemma 11 and the continuity of $f$ imply that the function $Z_{k}: \Omega \rightarrow \mathbb{R}$,

$$
Z_{k}:=f \circ\left(\frac{\sum_{j=1}^{n} \lambda_{j}^{Y_{j k}} p_{j} x_{j}}{\sum_{j=1}^{n} \lambda_{j}^{Y_{j k}} p_{j}}\right), \quad k \in \mathbb{N}_{+}
$$

is measurable, and therefore it is also a random variable. By applying Lemma 4 with $l_{i}:=i$ $(1 \leq i \leq n-1), f$ is bounded on the convex hull of the set $\left\{x_{1}, \ldots, x_{n}\right\}$. It follows that $\left(Z_{k}\right)_{k=1}^{\infty}$ is also bounded, and thus $Z_{k}\left(k \in \mathbb{N}_{+}\right)$is $P$-integrable. By (17),

$$
E\left(Z_{k}\right)=\int_{\Omega} Z_{k} d P=D_{1 k}, \quad k \in \mathbb{N}_{+}
$$

It is a consequence of Lebesgue's convergence theorem that

$$
\lim _{k \rightarrow \infty} D_{1 k}=\int_{\Omega} \lim _{k \rightarrow \infty} Z_{k} d P
$$


By the strong law of large numbers,

$$
\lim _{k \rightarrow \infty} \frac{Y_{j k}}{k}=q_{j} \quad P \text {-a.e., } 1 \leq j \leq n .
$$

Therefore, by Lemma 10,

$$
\begin{aligned}
& \lim _{k \rightarrow \infty}\left(\frac{Y_{j k}}{k} \ln \left(\lambda_{j}\right)-\frac{Y_{1 k}}{k} \ln \left(\lambda_{1}\right)\right) \\
& \quad=q_{j} \ln \left(\lambda_{j}\right)-q_{1} \ln \left(\lambda_{1}\right)<0 \quad P \text {-a.e., } 2 \leq j \leq n,
\end{aligned}
$$

and this leads to

$$
\begin{aligned}
\lim _{k \rightarrow \infty} \frac{\lambda_{j}^{Y_{j k}} p_{j}}{\lambda_{1}^{Y_{1 k}} p_{1}} & =\lim _{k \rightarrow \infty} \frac{p_{j}}{p_{1}} \exp \left(k\left(\frac{Y_{j k}}{k} \ln \left(\lambda_{j}\right)-\frac{Y_{1 k}}{k} \ln \left(\lambda_{1}\right)\right)\right) \\
& =0 \quad P \text {-a.e., } 2 \leq j \leq n .
\end{aligned}
$$

Consequently, we get from (18) that

$$
\lim _{k \rightarrow \infty} D_{1 k}=f\left(x_{1}\right)
$$

The proof is now complete.

The following lemma extends Lemma 14 in [4].

Lemma 12 Let $k \in \mathbb{N}$ and $\left(i_{1}, \ldots, i_{n}\right) \in S_{k+1}$ be fixed. If we set

$$
z\left(i_{1}, \ldots, i_{n}\right):=\left\{l \in\{1, \ldots, n\} \mid i_{l} \neq 0\right\}
$$

then

$$
\begin{aligned}
& \sum_{l \in z\left(i_{1}, \ldots, i_{n}\right)}\left(\frac{k !}{i_{1} ! \cdots i_{l-1} !\left(i_{l}-1\right) ! i_{l+1} ! \cdots i_{n} !} \frac{1}{\left(\lambda_{1}-1\right)^{i_{1}}} \cdots \frac{1}{\left(\lambda_{l-1}-1\right)^{i_{l-1}}}\right. \\
& \left.\cdot \frac{1}{\left(\lambda_{l}-1\right)^{i_{l}-1}} \frac{1}{\left(\lambda_{l+1}-1\right)^{i_{l+1}}} \cdots \frac{1}{\left(\lambda_{n}-1\right)^{i_{n}}} \frac{1}{\lambda_{l}-1}\right)=\frac{(k+1) !}{i_{1} ! \cdots i_{n} !} \prod_{j=1}^{n} \frac{1}{\left(\lambda_{j}-1\right)^{i_{j}}} .
\end{aligned}
$$

Proof The lowest common denominator is $i_{1} ! \cdots i_{n}$ !. Combined with $\sum_{j=1}^{n} i_{j}=k+1$, the result follows.

We can now prove the first main result.

Proof of Theorem 1 (a) The proof is divided into three steps.

I. Since $S_{0}=\{(0, \ldots, 0)\}$,

$$
D_{0}(\lambda)=\left(\sum_{j=1}^{n} \lambda_{j}^{0} p_{j}\right) f\left(\frac{\sum_{j=1}^{n} \lambda_{j}^{0} p_{j} x_{j}}{\sum_{j=1}^{n} \lambda_{j}^{0} p_{j}}\right)=f\left(\sum_{j=1}^{n} p_{j} x_{j}\right) .
$$


II. Next, we prove that $D_{k}(\lambda) \leq D_{k+1}(\lambda)(k \in \mathbb{N})$.

It is not hard to see that for every $\left(i_{1}, \ldots, i_{n}\right) \in S_{k}$,

$$
\begin{aligned}
\frac{\sum_{j=1}^{n} \lambda_{j}^{i_{j}} p_{j} x_{j}}{\sum_{j=1}^{n} \lambda_{j}^{i_{j}} p_{j}}= & \frac{1}{d(\lambda)+1} \cdot \sum_{l=1}^{n}\left(\frac{1}{\lambda_{l}-1} \cdot \frac{\sum_{j=1}^{n} \lambda_{j}^{i_{j}} p_{j} x_{j}+\left(\lambda_{l}-1\right) \lambda_{l}^{i_{l}} p_{l} x_{l}}{\sum_{j=1}^{n} \lambda_{j}^{i_{j}} p_{j}+\left(\lambda_{l}-1\right) \lambda_{l}^{i_{l}} p_{l}}\right. \\
& \left.\cdot \frac{\sum_{j=1}^{n} \lambda_{j}^{i_{j}} p_{j}+\left(\lambda_{l}-1\right) \lambda_{l}^{i_{l}} p_{l}}{\sum_{j=1}^{n} \lambda_{j}^{i_{j}} p_{j}}\right) .
\end{aligned}
$$

According to Theorem A, this yields that

$$
\begin{aligned}
f\left(\frac{\sum_{j=1}^{n} \lambda_{j}^{i_{j}} p_{j} x_{j}}{\sum_{j=1}^{n} \lambda_{j}^{i_{j}} p_{j}}\right) \leq & \frac{1}{d(\lambda)+1} \sum_{l=1}^{n}\left(\frac{1}{\lambda_{l}-1} \cdot \frac{\sum_{j=1}^{n} \lambda_{j}^{i_{j}} p_{j}+\left(\lambda_{l}-1\right) \lambda_{l}^{i_{l}} p_{l}}{\sum_{j=1}^{n} \lambda_{j}^{i_{j}} p_{j}}\right. \\
& \left.\cdot f\left(\frac{\sum_{j=1}^{n} \lambda_{j}^{i_{j}} p_{j} x_{j}+\left(\lambda_{l}-1\right) \lambda_{l}^{i_{l}} p_{l} x_{l}}{\sum_{j=1}^{n} \lambda_{j}^{i_{j}} p_{j}+\left(\lambda_{l}-1\right) \lambda_{l}^{i_{l}} p_{l}}\right)\right) .
\end{aligned}
$$

Consequently,

$$
\begin{aligned}
D_{k}(\lambda) \leq & \frac{1}{(d(\lambda)+1)^{k+1}} \sum_{\left(i_{1}, \ldots, i_{n}\right) \in S_{k}}\left(\frac { k ! } { i _ { 1 } ! \cdots i _ { n } ! } \prod _ { j = 1 } ^ { n } \frac { 1 } { ( \lambda _ { j } - 1 ) ^ { i _ { j } } } \sum _ { l = 1 } ^ { n } \left(\frac{1}{\lambda_{l}-1}\right.\right. \\
& \left.\left.\cdot\left(\sum_{j=1}^{n} \lambda_{j}^{i_{j}} p_{j}+\left(\lambda_{l}-1\right) \lambda_{l}^{i_{l}} p_{l}\right) f\left(\frac{\sum_{j=1}^{n} \lambda_{j}^{i_{j}} p_{j} x_{j}+\left(\lambda_{l}-1\right) \lambda_{l}^{i_{l}} p_{l} x_{l}}{\sum_{j=1}^{n} \lambda_{j}^{i_{j}} p_{j}+\left(\lambda_{l}-1\right) \lambda_{l}^{i_{l}} p_{l}}\right)\right)\right) .
\end{aligned}
$$

Bringing in Lemma 12, we find that the right-hand side of (21) can be written in the form

$$
\frac{1}{(d(\lambda)+1)^{k+1}} \cdot \sum_{\left(i_{1}, \ldots, i_{n}\right) \in S_{k+1}}\left(\frac{(k+1) !}{i_{1} ! \cdots i_{n} !} \prod_{j=1}^{n} \frac{1}{\left(\lambda_{j}-1\right)^{i_{j}}}\left(\sum_{j=1}^{n} \lambda_{j}^{i_{j}} p_{j}\right) f\left(\frac{\sum_{j=1}^{n} \lambda_{j}^{i_{j}} p_{j} x_{j}}{\sum_{j=1}^{n} \lambda_{j}^{i_{j}} p_{j}}\right)\right),
$$

which is just $D_{k+1}(\lambda)$.

III. Finally, we prove that

$$
D_{k}(\lambda) \leq \sum_{j=1}^{n} p_{j} f\left(x_{j}\right), \quad k \in \mathbb{N}_{+}
$$

It follows from Theorem A that

$$
\begin{aligned}
D_{k}(\lambda) \leq & \frac{1}{(d(\lambda)+1)^{k}} \sum_{\left(i_{1}, \ldots, i_{n}\right) \in S_{k}}\left(\frac{k !}{i_{1} ! \cdots i_{n} !} \prod_{j=1}^{n} \frac{1}{\left(\lambda_{j}-1\right)^{i_{j}}} \sum_{j=1}^{n} \lambda_{j}^{i_{j}} p_{j} f\left(x_{j}\right)\right) \\
= & \frac{1}{(d(\lambda)+1)^{k}} \sum_{j=1}^{n}\left(\sum_{\left(i_{1}, \ldots, i_{n}\right) \in S_{k}} \frac{k !}{i_{1} ! \cdots i_{n} !} \frac{1}{\left(\lambda_{1}-1\right)^{i_{1}}} \cdots \frac{1}{\left(\lambda_{j-1}-1\right)^{i_{j-1}}}\right. \\
& \left.\cdot \frac{\lambda_{j}^{i_{j}}}{\left(\lambda_{j}-1\right)^{i_{j}}} \frac{1}{\left(\lambda_{j+1}-1\right)^{i_{j+1}}} \cdots \frac{1}{\left(\lambda_{n}-1\right)^{i_{n}}}\right) p_{j} f\left(x_{j}\right), \quad k \in \mathbb{N}_{+} .
\end{aligned}
$$


The multinomial theorem shows that

$$
\begin{aligned}
& \sum_{\left(i_{1}, \ldots, i_{n}\right) \in S_{k}} \frac{k !}{i_{1} ! \cdots i_{n} !} \frac{1}{\left(\lambda_{1}-1\right)^{i_{1}}} \cdots \frac{1}{\left(\lambda_{j-1}-1\right)^{i_{j-1}}} \\
& \cdot \frac{\lambda_{j}^{i_{j}}}{\left(\lambda_{j}-1\right)^{i_{j}}} \frac{1}{\left(\lambda_{j+1}-1\right)^{i_{j+1}}} \cdots \frac{1}{\left(\lambda_{n}-1\right)^{i_{n}}}=(d(\lambda)+1)^{k}, \quad 1 \leq j \leq n,
\end{aligned}
$$

hence (24) implies (22).

(b) It is enough to confirm that for $l=1, \ldots, n$,

$$
\begin{aligned}
& \lim _{k \rightarrow \infty} p_{l} \frac{1}{(d(\lambda)+1)^{k}} \\
& \quad \cdot \sum_{\left(i_{1}, \ldots, i_{n}\right) \in S_{k}} \frac{k !}{i_{1} ! \cdots i_{n} !} \lambda_{l}^{i_{l}} \prod_{j=1}^{n} \frac{1}{\left(\lambda_{j}-1\right)^{i_{j}}} f\left(\frac{\sum_{j=1}^{n} \lambda_{j}^{i_{j}} p_{j} x_{j}}{\sum_{j=1}^{n} \lambda_{j}^{i_{j}} p_{j}}\right)=p_{l} f\left(x_{l}\right) .
\end{aligned}
$$

This is evident if $p_{l}=0$. If $p_{l}>0$, then we have it from Theorem 2 .

The proof is complete.

Proof of Theorem 3 It is enough to prove that there is strict inequality for a fixed $\left(i_{1}, \ldots, i_{n}\right) \in S_{k}(k \in \mathbb{N})$ in (20) and in (23), respectively.

The treatment of inequality (23) is pretty immediate. Under the conditions of the theorem,

$$
f\left(\frac{\sum_{j=1}^{n} \lambda_{j}^{i_{j}} p_{j} x_{j}}{\sum_{j=1}^{n} \lambda_{j}^{i_{j}} p_{j}}\right)<\frac{1}{\sum_{j=1}^{n} \lambda_{j}^{i_{j}} p_{j}} \sum_{j=1}^{n} \lambda_{j}^{i_{j}} p_{j} f\left(x_{j}\right), \quad\left(i_{1}, \ldots, i_{n}\right) \in S_{k}(k \in \mathbb{N})
$$

by Theorem C. It follows that the last inequality in (8) is strict (this is true for every $n \geq 2$ ).

It remains to study (20). Let $k \in \mathbb{N}$ be fixed, and let

$$
\left(i_{1}, \ldots, i_{n}\right)=(k, 0, \ldots, 0) \in S_{k} .
$$

Under the hypotheses, the coefficients of the vectors

$$
\frac{\sum_{j=1}^{n} \lambda_{j}^{i_{j}} p_{j} x_{j}+\left(\lambda_{l}-1\right) \lambda_{l}^{i_{l}} p_{l} x_{l}}{\sum_{j=1}^{n} \lambda_{j}^{i_{j}} p_{j}+\left(\lambda_{l}-1\right) \lambda_{l}^{i_{l}} p_{l}}, \quad 1 \leq l \leq n
$$

are positive in (19), and therefore by Theorem C, it is enough to prove that there exist two different vectors in (25).

We begin with the case $n=2$. Then $x_{1} \neq x_{2}$, and (25) consists of two vectors, namely

$$
v_{1}:=\frac{\lambda_{1}^{k+1} p_{1} x_{1}+p_{2} x_{2}}{\lambda_{1}^{k+1} p_{1}+p_{2}}, \quad v_{2}:=\frac{\lambda_{1}^{k} p_{1} x_{1}+\lambda_{2} p_{2} x_{2}}{\lambda_{1}^{k} p_{1}+\lambda_{2} p_{2}} .
$$

Elementary considerations show that $v_{1}=v_{2}$ implies that

$$
\lambda_{1}^{k} p_{1} p_{2}\left(1-\lambda_{1} \lambda_{2}\right) x_{1}=\lambda_{1}^{k} p_{1} p_{2}\left(1-\lambda_{1} \lambda_{2}\right) x_{2},
$$

and thus $x_{1}=x_{2}$, which is a contradiction. 
Now, we continue with the case $n=3$. Then the following three vectors belong to (25):

$$
\begin{aligned}
& v_{1}:=\frac{\lambda_{1}^{k+1} p_{1} x_{1}+p_{2} x_{2}+p_{3} x_{3}}{\lambda_{1}^{k+1} p_{1}+p_{2}+p_{3}}, \quad v_{2}:=\frac{\lambda_{1}^{k} p_{1} x_{1}+\lambda_{2} p_{2} x_{2}+p_{3} x_{3}}{\lambda_{1}^{k} p_{1}+\lambda_{2} p_{2}+p_{3}} \\
& v_{3}:=\frac{\lambda_{1}^{k} p_{1} x_{1}+p_{2} x_{2}+\lambda_{3} p_{3} x_{3}}{\lambda_{1}^{k} p_{1}+\lambda_{2} p_{2}+\lambda_{3} p_{3}}
\end{aligned}
$$

Suppose $v_{1}=v_{2}=v_{3}$. A simple but troublesome calculation confirms that

$$
\begin{aligned}
& \lambda_{1}^{k} p_{1} p_{2}\left(\lambda_{1}-1\right)\left(x_{1}-x_{2}\right)+\lambda_{1}^{k} p_{1} p_{3}\left(\lambda_{1} \lambda_{3}-1\right)\left(x_{1}-x_{3}\right) \\
& \quad+p_{2} p_{3}\left(\lambda_{3}-1\right)\left(x_{2}-x_{3}\right)=0
\end{aligned}
$$

and

$$
\begin{aligned}
& \lambda_{1}^{k} p_{1} p_{2}\left(\lambda_{1} \lambda_{2}-1\right)\left(x_{1}-x_{2}\right)+\lambda_{1}^{k} p_{1} p_{3}\left(\lambda_{1}-1\right)\left(x_{1}-x_{3}\right) \\
& \quad+p_{2} p_{3}\left(1-\lambda_{2}\right)\left(x_{2}-x_{3}\right)=0 .
\end{aligned}
$$

Therefore, recalling that

$$
x_{1}-x_{3}=\left(x_{1}-x_{2}\right)+\left(x_{2}-x_{3}\right)
$$

we have a homogeneous system of two linear equations with solutions $x_{1}-x_{2}$ and $x_{2}-x_{3}$. A few easy calculations yield that the determinant of the matrix of this system is

$$
\lambda_{1}^{k} p_{1} p_{2} p_{3}\left(\lambda_{1}^{k+1} p_{1}+p_{2}+p_{3}\right)\left(\lambda_{1}+\lambda_{2}+\lambda_{3}-\lambda_{1} \lambda_{2} \lambda_{3}-2\right)
$$

$\lambda_{i}>1(i=1,2,3)$ implies that

$$
\lambda_{1}+\lambda_{2}+\lambda_{3}-\lambda_{1} \lambda_{2} \lambda_{3}-2<0
$$

and hence (26) is negative (it is only important that different from 0 ). From this we get

$$
x_{1}-x_{2}=x_{2}-x_{3}=0 \text {, }
$$

that is, $x_{1}=x_{2}=x_{3}$, which is also a contradiction.

The proof is complete.

Proof of Lemma 4 (a) It is well known (see [11]) that a convex function on an open convex set in $\mathbb{R}^{m}$ is continuous.

(b) $h_{l_{1}, \ldots, l_{m}}$ is bounded above since discrete Jensen's inequality shows

$$
\begin{aligned}
h_{l_{1}, \ldots, l_{m}}\left(t_{1}, \ldots, t_{m}\right) & =f\left(\sum_{i=1}^{m} t_{i} x_{l_{i}}+\left(1-\sum_{i=1}^{m} t_{i}\right) x_{l}\right) \\
& \leq \sum_{i=1}^{m} t_{i} f\left(x_{l_{i}}\right)+\left(1-\sum_{i=1}^{m} t_{i}\right) f\left(x_{l}\right) \\
& \leq \max \left(f\left(x_{l_{1}}\right), \ldots, f\left(x_{l_{m}}\right), f\left(x_{l}\right)\right), \quad\left(t_{1}, \ldots, t_{m}\right) \in G_{m} .
\end{aligned}
$$


Let $\left(s_{1}, \ldots, s_{m}\right) \in \operatorname{int}\left(G_{m}\right)$ be fixed. Since $h_{l_{1}, \ldots, l_{m}}$ is convex, there is a linear functional $L$ on $\mathbb{R}^{m}$ (a support of $f$ at $\left(s_{1}, \ldots, s_{m}\right)$ ) such that

$$
\begin{aligned}
h_{l_{1}, \ldots, l_{m}}\left(t_{1}, \ldots, t_{m}\right) \geq & h_{l_{1}, \ldots, l_{m}}\left(s_{1}, \ldots, s_{m}\right) \\
& +L\left(t_{1}-s_{1}, \ldots, t_{m}-s_{m}\right), \quad\left(t_{1}, \ldots, t_{m}\right) \in \operatorname{int}\left(G_{m}\right),
\end{aligned}
$$

and therefore the continuity of $L$ on the compact set $\left(s_{1}, \ldots, s_{m}\right)+G_{m}$ yields that $h_{l_{1}, \ldots, l_{m}}$ is bounded below on $\operatorname{int}\left(G_{m}\right)$. It is easy to check that inequality (27) holds for every boundary point of $G_{m}$, too.

(c) Because $G_{m}$ is a polytope, this follows from (b) (see [12]).

The proof is complete.

Proof of Theorem 5 (a) We can obviously suppose that $l=n$.

An elementary calculation gives

$$
\begin{aligned}
& \lim _{\lambda_{n} \rightarrow 1_{+}} \frac{1}{(d(\lambda)+1)^{k}} \prod_{j=1}^{n} \frac{1}{\left(\lambda_{j}-1\right)^{i_{j}}} \\
& \quad=\lim _{\lambda_{1} \rightarrow 1_{+}} \prod_{j=1}^{n} \frac{1}{\left(\lambda_{j}+\sum_{\substack{p=1 \\
p \neq j}}^{n} \frac{\lambda_{j}-1}{\lambda_{p}-1}\right)^{i_{j}}} \\
& \quad=\left\{\begin{array}{ll}
1 & \text { if } i_{n}=k, \\
0 & \text { if } i_{n} \in\{0, \ldots, k-1\},
\end{array} \quad\left(i_{1}, \ldots, i_{n}\right) \in S_{k} .\right.
\end{aligned}
$$

If $p_{n}=0$, then for every $\left(i_{1}, \ldots, i_{n}\right) \in S_{k}$,

$$
\lim _{\lambda_{n} \rightarrow 1+}\left(\sum_{j=1}^{n} \lambda_{j}^{i_{j}} p_{j}\right) f\left(\frac{\sum_{j=1}^{n} \lambda_{j}^{i_{j}} p_{j} x_{j}}{\sum_{j=1}^{n} \lambda_{j}^{i_{j}} p_{j}}\right)=\left(\sum_{j=1}^{n-1} \lambda_{j}^{i_{j}} p_{j}\right) f\left(\frac{\sum_{j=1}^{n-1} \lambda_{j}^{i_{j}} p_{j} x_{j}}{\sum_{j=1}^{n-1} \lambda_{j}^{i_{j}} p_{j}}\right),
$$

and therefore, by (28)

$$
\lim _{\lambda_{n} \rightarrow 1+} D_{k}(\lambda)=f\left(\sum_{j=1}^{n-1} p_{j} x_{j}\right)
$$

which we wanted.

In the rest of the proof, $0<p_{n}<1$. From this, the existence of an integer $1 \leq m \leq n-1$ such that $m$ elements of the sequence $p_{1}, \ldots, p_{n-1}$ belong to the open interval $] 0,1[$ and $n-1-m$ members are 0 follows. We can assume that $\left.p_{1}, \ldots, p_{m} \in\right] 0,1\left[\right.$ and $p_{m+1}=\cdots=$ $p_{n-1}=0$.

Consider the function $h_{1, \ldots, m}$ defined in (10).

Fix $\left(i_{1}, \ldots, i_{n}\right) \in S_{k}$. Then

$$
\lim _{\lambda_{n} \rightarrow 1+} \frac{\lambda_{j}^{i_{j}} p_{j}}{\sum_{j=1}^{n} \lambda_{j}^{i_{j}} p_{j}}=\lim _{\lambda_{n} \rightarrow 1+} \begin{cases}\frac{\lambda_{j}^{i_{j}} p_{j}}{\sum_{j=1}^{m} \lambda_{j}^{i_{j}} p_{j}+p_{n}}>0 & \text { if } 1 \leq j \leq m, \\ \frac{p_{n}}{\sum_{j=1}^{m} \lambda_{j}^{i_{j}} p_{j}+p_{n}}>0 & \text { if } j=n,\end{cases}
$$


which shows that

$$
\lim _{\lambda_{n} \rightarrow 1+}\left(\frac{\lambda_{1}^{i_{1}} p_{1}}{\sum_{j=1}^{n} \lambda_{j}^{i_{j}} p_{j}}, \ldots, \frac{\lambda_{m}^{i_{m}} p_{m}}{\sum_{j=1}^{n} \lambda_{j}^{i_{j}} p_{j}}\right)=\left(\frac{\lambda_{1}^{i_{1}} p_{1}}{\sum_{j=1}^{m} \lambda_{j}^{i_{j}} p_{j}+p_{n}}, \ldots, \frac{\lambda_{m}^{i_{m}} p_{m}}{\sum_{j=1}^{m} \lambda_{j}^{i_{j}} p_{j}+p_{n}}\right)
$$

is an interior point of $G_{m}$. In light of the continuity of $h_{1, \ldots, m}$ on $\operatorname{int}\left(G_{m}\right)$ (see Lemma 4), this implies that

$$
\begin{aligned}
& \lim _{\lambda_{n} \rightarrow 1_{+}}\left(\sum_{j=1}^{n} \lambda_{j}^{i_{j}} p_{j}\right) f\left(\frac{\sum_{j=1}^{n} \lambda_{j}^{i_{j}} p_{j} x_{j}}{\sum_{j=1}^{n} \lambda_{j}^{i_{j}} p_{j}}\right) \\
& \quad=\lim _{\lambda_{n} \rightarrow 1+}\left(\sum_{j=1}^{m} \lambda_{j}^{i_{j}} p_{j}+\lambda_{n}^{i_{n}} p_{n}\right) h\left(\frac{\lambda_{1}^{i_{1}} p_{1}}{\sum_{j=1}^{n} \lambda_{j}^{i_{j}} p_{j}}, \ldots, \frac{\lambda_{m}^{i_{m}} p_{m}}{\sum_{j=1}^{n} \lambda_{j}^{i_{j}} p_{j}}\right) \\
& \quad=\left(\sum_{j=1}^{m} \lambda_{j}^{i_{j}} p_{j}+p_{n}\right) h\left(\frac{\lambda_{1}^{i_{1}} p_{1}}{\sum_{j=1}^{m} \lambda_{j}^{i_{j}} p_{j}+p_{n}}, \ldots, \frac{\lambda_{m}^{i_{m}} p_{m}}{\sum_{j=1}^{m} \lambda_{j}^{i_{j}} p_{j}+p_{n}}\right) .
\end{aligned}
$$

The limit relations (28) and (29) entail

$$
\begin{aligned}
& \lim _{\lambda_{n} \rightarrow 1+} \frac{1}{(d(\lambda)+1)^{k}} \prod_{j=1}^{n} \frac{1}{\left(\lambda_{j}-1\right)^{i_{j}}}\left(\sum_{j=1}^{n} \lambda_{j}^{i_{j}} p_{j}\right) f\left(\frac{\sum_{j=1}^{n} \lambda_{j}^{i_{j}} p_{j} x_{j}}{\sum_{j=1}^{n} \lambda_{j}^{i_{j}} p_{j}}\right) \\
& \quad= \begin{cases}f\left(\sum_{j=1}^{m} p_{j} x_{j}+p_{n} x_{n}\right) & \text { if } i_{n}=k, \\
0 & \text { if } i_{n} \in\{0, \ldots, k-1\},\end{cases}
\end{aligned}
$$

which gives the result.

We begin the proof of the second part of the result with some limit formulas.

It is obvious that

$$
\lim _{\lambda_{l} \rightarrow \infty} \frac{1}{(d(\lambda)+1)^{k}}=\frac{1}{\left(\sum_{\substack{j=1 \\ j \neq l}}^{n} \frac{1}{\lambda_{j}-1}+1\right)^{k}} .
$$

It is also easy to calculate that for each $\left(i_{1}, \ldots, i_{n}\right) \in S_{k}$,

$$
\lim _{\lambda_{l} \rightarrow \infty} \prod_{j=1}^{n} \frac{1}{\left(\lambda_{j}-1\right)^{i_{j}}}\left(\sum_{j=1}^{n} \lambda_{j}^{i_{j}} p_{j}\right)= \begin{cases}\prod_{j=1}^{n} \frac{1}{\left(\lambda_{j}-1\right)^{i_{j}}}\left(\sum_{j=1}^{n} \lambda_{j}^{i_{j}} p_{j}\right), & i_{l}=0, \\ \frac{p_{l}}{\left.\prod_{\substack{j=1 \\ j \neq l}}^{n} \lambda_{j}-1\right)^{i_{j}}}, & i_{l} \neq 0 .\end{cases}
$$

$\left(b_{1}\right)$ The expression

$$
f\left(\frac{\sum_{j=1}^{n} \lambda_{j}^{i_{j}} p_{j} x_{j}}{\sum_{j=1}^{n} \lambda_{j}^{i_{j}} p_{j}}\right)
$$

does not depend on $\lambda_{l}$ for each $\left(i_{1}, \ldots, i_{n}\right) \in S_{k}$, from which we have (11) by (30) and (31).

$\left(\mathrm{b}_{2}\right)$ If $i_{l}=0$, then (32) is independent of $\lambda_{l}$, hence we can have the coefficient in (12) and the first member in the sum (13) from (30) and (31).

Suppose $\left(i_{1}, \ldots, i_{n}\right) \in S_{k}$ such that $i_{l} \neq 0$. 
By using (31), it remains to study the existence of the limit of (32) as $\lambda_{l} \rightarrow \infty$. Under the conditions on the zero elements of the sequence $p_{1}, \ldots, p_{n}$, we have to consider

$$
\lim _{\lambda_{l} \rightarrow \infty} h_{l_{1}, \ldots, l_{m}}\left(\frac{\lambda_{l_{1}}^{i_{l_{1}}} p_{l_{1}}}{\sum_{j=1}^{m} \lambda_{l_{j}}^{i_{l_{j}}} p_{l_{j}}+\lambda_{l}^{i_{l}} p_{l}}, \ldots, \frac{\lambda_{l_{m}}^{i_{l_{m}}} p_{l_{m}}}{\sum_{j=1}^{m} \lambda_{l_{j}}^{i_{l_{j}}} p_{l_{j}}+\lambda_{l}^{i_{l}} p_{l}}\right)
$$

Since

$$
\lim _{\lambda_{l} \rightarrow \infty}\left(\frac{\lambda_{l_{1}}^{i_{l_{1}}} p_{l_{1}}}{\sum_{j=1}^{m} \lambda_{l_{j}}^{i_{l_{j}}} p_{l_{j}}+\lambda_{l}^{i_{l}} p_{l}}, \ldots, \frac{\lambda_{l_{m}}^{i_{l_{m}}} p_{l_{m}}}{\sum_{j=1}^{m} \lambda_{l_{j}}^{i_{l_{j}}} p_{l_{j}}+\lambda_{l}^{i_{l}} p_{l}}\right)=(0, \ldots, 0)
$$

and

$$
\left(\frac{\lambda_{l_{1}}^{i_{l_{1}}} p_{l_{1}}}{\sum_{j=1}^{m} \lambda_{l_{j}}^{i_{l_{j}}} p_{l_{j}}+\lambda_{l}^{i_{l}} p_{l}}, \ldots, \frac{\lambda_{l_{m}}^{i_{l_{m}}} p_{l_{m}}}{\sum_{j=1}^{m} \lambda_{l_{j}}^{i_{l_{j}}} p_{l_{j}}+\lambda_{l}^{i_{l}} p_{l}}\right)
$$

is an interior point of the domain $G_{m}$ of $h_{l_{1}, \ldots, l_{m}}$ for every $\lambda_{l}>1$, Lemma 4(c) implies that the limit (33) exists and it is $\bar{h}_{l_{1}, \ldots, l_{m}}(0, \ldots, 0)$. According to this and (31)

$$
\lim _{\lambda_{l} \rightarrow \infty} \prod_{j=1}^{n} \frac{1}{\left(\lambda_{j}-1\right)^{i_{j}}}\left(\sum_{j=1}^{n} \lambda_{j}^{i_{j}} p_{j}\right) f\left(\frac{\sum_{j=1}^{n} \lambda_{j}^{i_{j}} p_{j} x_{j}}{\sum_{j=1}^{n} \lambda_{j}^{i_{j}} p_{j}}\right)=\frac{p_{l} \bar{h}_{l_{1}, \ldots, l_{m}}(0, \ldots, 0)}{\prod_{\substack{j=1 \\ j \neq l}}^{n}\left(\lambda_{j}-1\right)^{i_{j}}},
$$

showing that

$$
\begin{aligned}
& \lim _{\lambda_{l} \rightarrow \infty} \sum_{\substack{\left(i_{1}, \ldots, i_{n}\right) \in S_{k} \\
i_{l} \neq 0}} \frac{k !}{i_{1} ! \cdots i_{n} !} \prod_{j=1}^{n} \frac{1}{\left(\lambda_{j}-1\right)^{i_{j}}}\left(\sum_{j=1}^{n} \lambda_{j}^{i_{j}} p_{j}\right) f\left(\frac{\sum_{j=1}^{n} \lambda_{j}^{i_{j}} p_{j} x_{j}}{\sum_{j=1}^{n} \lambda_{j}^{i_{j}} p_{j}}\right) \\
& =p_{l} \bar{h}_{l_{1}, \ldots, l_{m}}(0, \ldots, 0) \sum_{\substack{\left(i_{1}, \ldots, i_{n}\right) \in S_{k} \\
i_{l} \neq 0}} \frac{k !}{i_{1} ! \cdots i_{n} !} \frac{1}{\prod_{\substack{j=1 \\
j \neq l}}^{n}\left(\lambda_{j}-1\right)^{i_{j}}} \\
& =p_{l} \bar{h}_{l_{1}, \ldots, l_{m}}(0, \ldots, 0) \cdot \sum_{i_{l}=1}^{k}\left(\frac{\left(k-i_{l}+1\right) \cdots k}{i_{l} !}\right. \\
& \left.\cdot \sum_{i_{1}+\cdots+i_{l-1}+i_{l+1}+\cdots+i_{n}=k-i_{l}} \frac{\left(k-i_{l}\right) !}{i_{1} ! \cdots i_{l-1} ! i_{l+1} ! \cdots i_{n} !} \frac{1}{\prod_{\substack{j=1 \\
j \neq l}}^{n}\left(\lambda_{j}-1\right)^{i_{j}}}\right) \\
& =p_{l} \bar{h}_{l_{1}, \ldots, l_{m}}(0, \ldots, 0) \sum_{i_{l}=1}^{k}\left(\begin{array}{c}
k \\
k-i_{l}
\end{array}\right)\left(\sum_{j=1} \frac{1}{\lambda_{j}-1}\right)^{k-i_{l}} \\
& =p_{l} \bar{h}_{l_{1}, \ldots, l_{m}}(0, \ldots, 0)\left(\left(\sum_{j=1}^{n} \frac{1}{\lambda_{j}-1}+1\right)^{k}-\left(\sum_{j=1}^{n} \frac{1}{\lambda_{j}-1}\right)^{k}\right) . \\
& j \neq l \\
& j \neq l
\end{aligned}
$$

We have got the second sum in (13).

The proof is complete. 


\section{Competing interests}

The author declares that they have no competing interests.

\section{Acknowledgements}

The author thanks the anonymous referees for their careful reading and valuable comments. This work was supported by Hungarian National Foundations for Scientific Research Grant No. K101217.

Received: 10 June 2013 Accepted: 23 October 2013 Published: 22 Nov 2013

\section{References}

1. Hardy, GH, Littlewood, JE, Pólya, G: Inequalities. Cambridge Mathematical Library Series. Cambridge University Press, Cambridge (1967)

2. Dragomir, SS: A refinement of Jensen's inequality with applications for $f$-divergence measures. Taiwan. J. Math. 14(1), 153-164 (2010)

3. Dragomir, SS: A new refinement of Jensen's inequality in linear spaces with applications. Math. Comput. Model. 52, 1497-1505 (2010)

4. Horváth, L: A parameter dependent refinement of the discrete Jensen's inequality for convex and mid-convex functions. J. Inequal. Appl. 2011, 26 (2011)

5. Horváth, L: A method to refine the discrete Jensen's inequality for convex and mid-convex functions. Math. Comput. Model. 54, 2451-2459 (2011)

6. Horváth, L, Pečarić, J: A refinement of the discrete Jensen's inequality. Math. Inequal. Appl. 14(4), 777-791 (2011)

7. Horváth, L, Khan, KA, Pečarić, J: On parameter dependent refinement of discrete Jensen's inequality for operator convex functions. J. Math. Comput. Sci. 2(3), 656-672 (2012)

8. Xiao, Z-G, Srivastava, HM, Zhang, Z-H: Further refinements of the Jensen inequalities based upon samples with repetitions. Math. Comput. Model. 51, 592-600 (2010)

9. Xia, Y: A refinement of Jensen's inequality for a class of increasing and concave functions. J. Inequal. Appl. 2008 Article ID 717614 (2008)

10. Mitrinović, DS, Pečarić, JE, Fink, AM: Classical and New Inequalities in Analysis. Mathematics and Its Applications, vol. 61. Kluwer Academic, Dordrecht (1993)

11. Niculescu, C, Persson, LE: Convex Functions and Their Applications: A Contemporary Approach. CMS Books in Mathematics. Springer, New York (2006)

12. Gale, D, Klee, V, Rockafellar, RT: Convex functions on convex polytopes. Proc. Am. Math. Soc. 19, $867-873$ (1968)

10.1186/1029-242X-2013-551

Cite this article as: Horváth: A new refinement of discrete Jensen's inequality depending on parameters. Journal of Inequalities and Applications 2013, 2013:551

\section{Submit your manuscript to a SpringerOpen ${ }^{\circ}$ journal and benefit from:}

- Convenient online submission

Rigorous peer review

- Immediate publication on acceptance

- Open access: articles freely available online

- High visibility within the field

- Retaining the copyright to your article 